\section{Blastic Epstein-Barr virus associated post-transplant lymphoproliferative disorder after allogeneic stem cell transplantation for severe aplastic anemia}

\author{
Masaaki Hotta, Aya Nakaya, Shinya \\ Fujita, Atsushi Satake, \\ Takahisa Nakanishi, Yoshiko Azuma, \\ Yukie Tsubokura, Akiko Konishi, \\ Hideaki Yoshimura, Tomoki Ito, \\ Kazuyoshi Ishii, Shosaku Nomura \\ First Department of Internal Medicine, \\ Kansai Medical University, Hirakata, \\ Japan
}

\begin{abstract}
Post-transplant lymphoproliferative disorder (PTLD) is a well-recognized complication of organ transplantation. Progress has recently been made in the pathological classification of PTLD. However, the clinical course has not been clarified because of the rarity of this disease. We experienced a case of PTLD with a fulminant clinical course. The patient had been under longterm immunosuppressive treatment for aplastic anemia. He received related allogeneic hematopoietic stem cell transplantation. Soon after transplantation, he developed PTLD. According to the guidelines, we reduced immunosuppression. However, the disease course was so fulminant that there was no time for the patient to respond, and he died of multi-organ failure. There may be various clinical types of PTLD, which may include some fulminant cases. In such a case, it is not sufficient to reduce immunosuppression. The patient should be carefully observed and an appropriate individual treatment should be chosen.
\end{abstract}

\section{Case Report}

A 31-year-old man was admitted to our hospital to receive related allogeneic hematopoietic stem cell transplantation (HSCT). He was diagnosed with mild aplastic anemia (AA) when he was 9 years old. Subsequently, his condition repeatedly oscillated from better to worse and he was given immunosuppression intermittently. $\mathrm{He}$ had previously been treated with antithymocyte globulin (ATG) and cyclosporine (CSA). More recently, his condition deteriorated; allogeneic HSCT was therefore planned. His older brother proved to be an 8/8 HLA-matched donor. Before transplantation, laboratory examination showed that his white blood cell count was $1700 / \mu \mathrm{L}$, hemoglobin was $5.9 \mathrm{~g} / \mathrm{dL}$, platelet count was $10,000 / \mu \mathrm{L}$, and reticulocyte count was $36,960 \mathrm{~g} / \mathrm{L}$. Bone marrow examination showed a total nucleated cell count of $27,000 / \mu \mathrm{L}$. Expression of CD55 and CD59 on granulocytes was within the reference range. He was diagnosed as stage 3 aplastic anemia. A conditioning regimen was administered consisting of $120 \mathrm{mg} / \mathrm{m}^{2}$ fludarabine, $9 \mathrm{mg} / \mathrm{m}^{2}$ rabbit ATG, 100 $\mathrm{mg} / \mathrm{kg}$ cyclophosphamide with continuous CSA infusion, and $10 \mathrm{mg} / \mathrm{m}^{2}$ methotrexate on days $+1,3,6$ and 11 as graft-versus-host disease (GVHD) prophylaxis. While receiving the conditioning regimen, there were no grade $\geq 3$ adverse events other than mucosal erosion and hematological toxicities. Neutrophil engraftment was achieved on day 17 . From day 30 , he developed highgrade fever and grade 2 skin GVHD. We started prednisolone $(1 \mathrm{~g} / \mathrm{kg})$ as a GVHD treatment. However, his fever was uncontrolled. At the same time, abnormal lymphocytes appeared in the peripheral blood examination. On day 35 , he developed sudden hepatomegaly and cervical lymphadenopathy. Liver biopsy was performed. Histopathological analysis showed diffuse large B cell lymphoma with Epstein-Barr Virus (EBV)-encoded small nuclear RNA positivity. The EBV-DNA level was $8.2 \times 10^{5}$ copies. We considered that he had developed post-transplant lymphoproliferative disorder (PTLD). His immunosuppression and prednisolone were stopped. However, high fever persisted and he died of multi-organ failure on day 40 (see Figure 1 for the course of HSTC).

\section{Discussion and Conclusions}

PTLD is a well-recognized complication of both solid organ transplantation and HSCT. In most cases, PTLD is associated with EBV infection, either as a consequence of post-transplantation viral reactivation or of primary EBV infection. Approximately $70 \%$ of PTLD cases are associated with EBV, however, the impact of EBV on survival is not clear. ${ }^{1}$ EBV positivity has been reported to be a poor prognostic factor, while non-EBV PTLD has also been seen to have a poor prognosis. ${ }^{2,3}$ In the updated 2016 World Health Organization lymphoma classification, PTLD is subclassified as plasmacytic hyperplasia PTLD, infectious mononucleosis PTLD, florid follicular hyperplasia PTLD, polymorphic PTLD,
Correspondence: Aya Nakaya, First Department of Internal Medicine, Kansai Medical University, 2-5-1, Shin-machi, Hirakata, Osaka 573-1010, Japan.

Tel.: +81.72.804.2503.

E-mail: nakaya1016@yahoo.co.jp

Key words: post-transplant lymphoproliferative disorder, allogeneic hematopoietic stem cell transplantation, aplastic anemia, EpsteinBarr Virus.

Contributions: MH, AN, SF, AS, TN, YT, YA, AK, HY, KI, TI, conception and design of the study, or acquisition of data, or analysis and interpretation of data; $\mathrm{MH}, \mathrm{AN}, \mathrm{SN}$, drafting the article or revising it critically for important intellectual content; AN, SN, final approval of the version to be submitted.

Conflict of interest: the authors declare no potential conflict of interest.

Funding: none.

Received for publication: 9 December 2017. Accepted for publication: 15 May 2018.

This work is licensed under a Creative Commons Attribution-NonCommercial 4.0 International License (CC BY-NC 4.0).

CCopyright M. Hotta et al., 2018

Licensee PAGEPress, Italy

Hematology Reports 2018; 10:7527

doi:10.4081/hr.2018.7527

monomorphic PTLD (B- and T-/NK-cell types) or classical Hodgkin lymphoma PTLD. ${ }^{4}$ Diffuse large B-cell lymphoma accounts for the majority of PTLD cases.

Risk factors for PTLD are HLA-mismatch between donor and recipient, conditioning regimens that include ATG, and Tcell-depleted transplants. ${ }^{5,6}$ EBV-DNA load is elevated exponentially in patients with PTLD, and weekly screening of the EBVDNA level is recommended for at least 3 months in high-risk patients who have undergone HSCT. ${ }^{7-9}$

The current case was at high risk of developing PTLD because of his long-term use of immunosuppression and previous use of ATG. The one point we regret was that we did not check his EBV-DNA level before transplantation. However, even if we had followed EBV-DNA levels during the treatment course, we might not have been able to predict the PTLD. The current case showed rapid onset and fulminant blastic disease. The fulminant form of PTLD is very uncommon and occurs in approximately $1 \%$ of PTLD patients. ${ }^{10}$

Historically, the initial treatment for PTLD is reduction of immunosuppression 


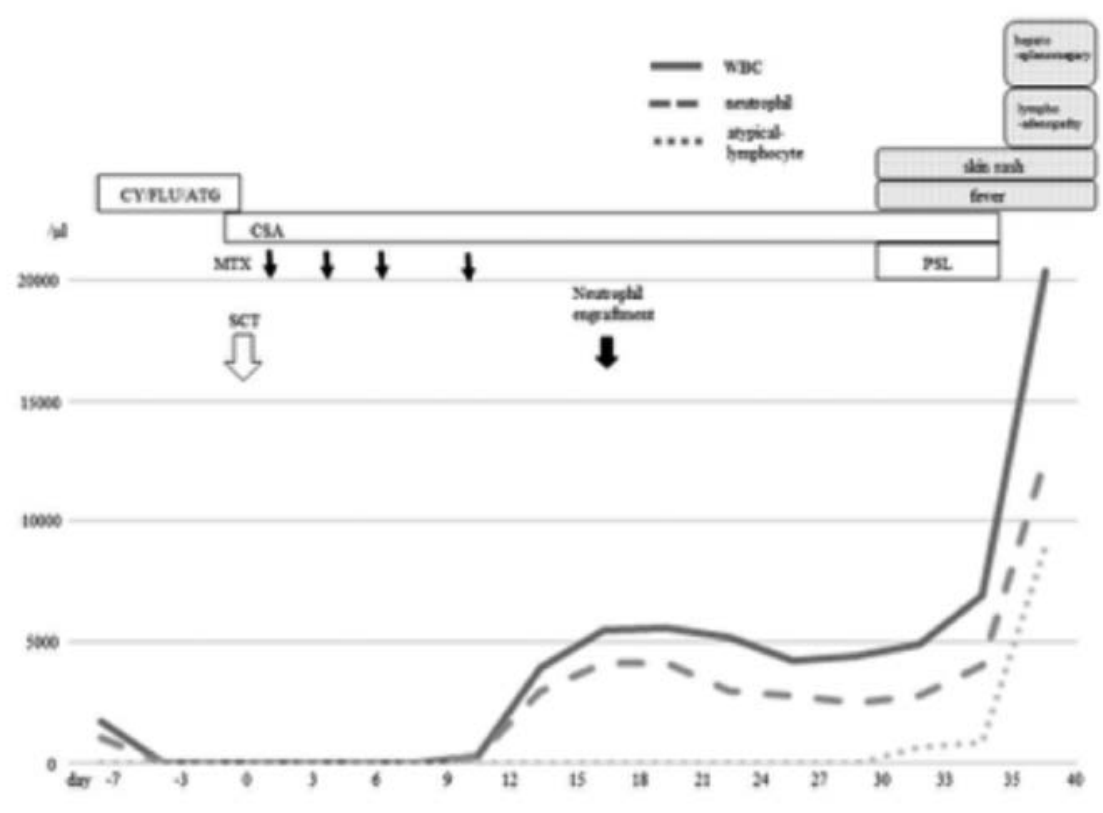

Figure 1. The course of hematopoietic stem cell transplantation. From day 30, he developed high-grade fever and grade 2 skin GVHD, and abnormal lymphocytes appeared in the peripheral blood examination. On day 35 , he developed sudden hepatomegaly and cervical lymphadenopathy, and he died on day 40. CY: cyclophosphamide; FLU: fludarabine; ATG: antithymocyte globulin; CSA: cyclosporine; MTX: methotrexate; SCT: stem cell transplantation; PSL: prednisolone; WBC: white cell blood.

(RIS). Thus, we stopped immunosuppression immediately; however, the disease course was too rapid for this to provide a therapeutic effect. Rituximab has recently been used for the treatment of PTLD, with an overall response rate (ORR) of 44$100 \%$. ${ }^{11,12}$ Furthermore, R-CHOP (rituximab, cyclophosphamide, doxorubicin, vincristine and prednisolone) is currently the preferred regimen for B-cell PTLD, with an ORR of up to $90 \% .^{13,14}$ In our case, the reduction of immunosuppression was not sufficient. We should have given chemotherapy as soon as we suspected PTLD. The standard treatment for PTLD has not been established. Current guidelines recommend RIS to the lowest tolerated levels, based on PTLD type, stage, critical illness and allograft risk, followed by close monitoring for response and escalation of therapy in the event that RIS alone is not sufficient. ${ }^{15}$ Classification of the pathology associated with PTLD has progressed recently. However, the clinical course has not been clarified because of the rarity of this disease. There has been no report on incidence according to original disease. We assume that, even if the primary disease is benign (such as AA), but the patient is under long-term immunosuppressive therapy, then there might be a higher risk of developing PTLD rather than malignant disease. There may be various clinical types of PTLD and these may include some ful- minant cases. In such a case, it is not sufficient to reduce immunosuppression alone. Each case should be carefully observed and an appropriate individual treatment should be chosen.

\section{References}

1. Oton $\mathrm{AB}$, Wang $\mathrm{H}$, Leleu $\mathrm{X}$, et al. Clinical and pathological prognostic markers for survival in adult patients with post-transplant lymphoproliferative disorders in solid transplant. Leuk Lymphoma 2008;49:1738-44.

2. Leblond V, Dhedin N, Mamzer Bruneel $\mathrm{MF}$, et al. Identification of prognostic factors in 61 patients with posttransplantation lymphoproliferative disorders. J Clin Oncol 2001;19:772-8.

3. Kinch A, Baecklund E, Backlin C, et al. A population-based study of 135 lymphomas after solid organ transplantation: The role of Epstein-Barr virus, hepatitis $\mathrm{C}$ and diffuse large B-cell lymphoma subtype in clinical presentation and survival. Acta Oncol 2014;53:66979.

4. Swerdlow SH, Campo E, Pileri SA, et al. The 2016 revision of the World Health Organization classification of lymphoid neoplasms. Blood 2016;127:2375-90.
5. Heslop HE. How I treat EBV lymphoproliferation. Blood 2009; 114:4002-8.

6. Cohen JM, Cooper N, Chakrabarti S, et al. EBV-related disease following haematopoietic stem cell transplantation with reduced intensity conditioning. Leuk Lymphoma 2007;48:256-69.

7. Rooney CM, Loftin SK, Holladay MS, et al. Early identification of EpsteinBarr virus-associated post-transplantation lymphoproliferative disease. $\mathrm{Br} \mathrm{J}$ Haematol 1995;89:98-103.

8. Styczynski J, Reusser P, Einsele H, et al. Management of HSV, VZV and EBV infections in patients with hematological malignancies and after SCT: guidelines from the Second European Conference on Infections in Leukemia. Bone Marrow Transplant 2009;43:75770.

9. Kimura H, Ito Y, Suzuki R, Nishiyama Y. Measuring Epstein-Barr virus (EBV) load: the significance and application for each EBV-associated disease. Rev Med Virol 2008;18:305-19.

10. Swinnen LJ, Costanzo-Nordin MR, Fisher SG, et al. Increased incidence of lymphoproliferative disorder after immunosuppression with the monoclonal antibody OKT3 in cardiac-transplant recipients. $\mathrm{N}$ Engl $\mathrm{J}$ Med 1990;323:1723-8.

11. Oertel SH, Verschuuren E, Reinke P, et al. Effect of anti-CD 20 antibody rituximab in patients with post-transplant lymphoproliferative disorder (PTLD). Am J Transplant 2005;5:2901-6.

12. Blaes AH, Peterson BA, Bartlett N, et al. Rituximab therapy is effective for posttransplant lymphoproliferative disorders after solid organ transplantation: results of a phase II trial. Cancer 2005;104:1661-7.

13. Choquet S, Trappe R, Leblond V, et al. CHOP-21 for the treatment of posttransplant lymphoproliferative disorders (PTLD) following solid organ transplantation. Haematologica 2007; 92:273-4

14. Trappe R, Oertel S, Leblond V, et al. Sequential treatment with rituximab followed by CHOP chemotherapy in adult B-cell post-transplant lymphoproliferative disorder (PTLD): the prospective international multicentre phase 2 PTLD-1 trial. Lancet Oncol 2012;13: 196-206.

15. Parker A, Bowles K, Bradley JA, et al. Management of post-transplant lymphoproliferative disorder in adult solid organ transplant recipients - BCSH and BTS Guidelines. Br J Haematol 2010;149:693-705. 